\title{
Effects of insoluble nano-particles on nanofluid droplet evaporation
}

\author{
Yan Wei ${ }^{1}$, Weiwei Deng ${ }^{2}$, and Ruey-Hung Chen ${ }^{3, *}$ \\ ${ }^{1}$ Department of Mechanical and Aerospace Engineering, University of Central Florida, Orlando, \\ FL 32816 \\ ${ }^{2}$ Department of Mechanical Engineering, Virginia Polytechnic Institute and State University, \\ Blacksburg, VA 25061 \\ ${ }^{3}$ Department of Mechanical and Aerospace Engineering, New Mexico State University, Las \\ Cruces, NM 88003
}

\begin{abstract}
A model is presented for predicting the evaporation behavior of liquid droplets containing nanosized insoluble particles. The model is constructed based on, and supported by, the existing experimental result. Of particular interest are the evolution of droplet size, and evaporation rate constant prior to the shell formation (also called the first drying or evaporation stage), when the particles forms a compact shell at the droplet surface. As the droplet evaporation proceeds, increasingly more particles are "included" on its surface, reducing the effective liquid surface area for evaporation causing deviation from the classical $d^{2}$-law for pure fluid droplet evaporation. Peclet number $(\mathrm{Pe})$, a non-dimensional ratio of particle diffusion time to the droplet lifetime, and initial particle concentration $\left(Y_{\mathrm{vo}}\right)$ are shown to play a role in the evaporation behavior prior to shell formation. The model predicts that: (1) the deviation from the classical $d^{2}-$ law is increasingly significant with increased $P e$ and $Y_{\text {vo }}$; (2) the time to shell formation decreases, and shell diameter increases, with increased $P e$ and initial particle concentration $\left(Y_{\mathrm{vo}}\right)$. As a consequence, $Y_{\mathrm{vo}} \cdot P e<1.8$ is necessary for the first stage to exist at all. Within the limiting value, the combination of larger $P e$ and smaller $Y_{\text {vo }}$ produces larger hollow shells with low densities. For $Y_{\text {vo }} \cdot P e>1.8$ the shell forms instantly and evaporation occurs after liquid diffuses through, and wet, the shell. The effect of contact angle $(\theta)$ on the effective liquid surface area for evaporation is also discussed.
\end{abstract}

Key words - evaporation, nanofluid, nano-particles, Peclet number, $d^{2}-$ law

*Corresponding author, chenrh@nmsu.edu 


\section{Introduction}

Evaporation of liquid droplets finds a wide range of applications - pharmaceutical, food, spray drying, energy, and propulsion fuels among others. The evaporation in stagnant as well as flowing surrounding gases has been studied both experimentally and analytically [1-8]. Evaporation of pure liquid droplets in the diffusion-controlled regime is known to follow the so-called $d^{2}$-law,

$$
d^{2}=d_{0}^{2}-K t
$$

where $d$ and $d_{o}$ are the instantaneous and initial droplet diameters, respectively, and $K$ is the evaporation coefficient. That is, the evaporation rate is directly related to the surface area that absorbs heat through diffusion. Droplets in convective environment does not follow the $d^{2}$-law, as the temperature and flow fields cease to be spherically symmetric and the exact value of exponent becomes a function of parameters such as Reynolds, Grashof, and Nusselt numbers. In general, the droplet evaporates follows a $d^{n}$ relationship [9], with $n=2$ for a diffusion-controlled evaporation in quiescent environments. Multicomponent droplets also exhibit evaporation behaviors according to the $d^{2}$-law that are analogous to a batch distillation [10], with varying values of $K$ as evaporation proceeds through different stages.

Another type of multicomponent liquid droplets consist of those containing insoluble particles (see for example [11-13]). For these types of droplets, the initial (i.e., first stage) evaporation rate was found to correspond to that of pure liquid, followed by formation of the shell of particles [11]. The shell forms when the droplet surface is populated with solid particles and its size is assumed constant through the rest of the process [11-16]. The second period of evaporation commences after shell formation, where evaporation occurs when the liquid from within the shell reaches the surface of the shell through capillary action. The second stage comprises essentially of drying of the wet particle agglomerate. A conceptual characteristic drying curve (CDC) was proposed to describe the heat transfer phenomena throughout the entire drying process [14]. Some experimental evidence supports the constant shell diameter [11]. 
For the purpose of the present study, it is worth noting that the particle agglomerate at the completion of drying possesses a wide range of morphology, from shallow crust (i.e., a shell with void inside) to compact cluster. In a study of water droplets containing nano-sized alumina particles, the morphology of dried particle agglomerate depends on the initial volume fraction of the colloids in the droplet and the mobility of particles within the liquid [17]. Derkachov et al. [18] in a study of evaporation-driven aggregation of 200-nm particles found that the morphology of nanoparticle aggregates ranges from spherical shells to the solid-like microcrystals. Such a wide range of morphological variations is attributable to effects including evaporation rate for a given initial concentration investigate (1:1,000 polystyrene spheres in water). The detail morphology of the final particle aggregates involved detail study of contact mechanics [19] and is beyond the scope of this study.

To explain the vast morphological variations, Derkachov et al. performed a numerical simulation [18] to investigate the inclusion process (the capturing of nanoparticles on the regressing droplet surface). The results reveal that the particle density on the regressing surface increases with time while forming "islands." As a consequence, the surface area from which evaporation occurs might be smaller than that determined by the droplet geometry depending how wetting the liquid is (i.e., an effect of the contact angle). Once the shell forms, the only liquid surface area for evaporation would only consist of the interstices between the packed particles. In the second stage of drying, the liquid will have to be transported to the interstitial regions on particle-populated surface through capillary action.

From the above results, one can reasonably hypothesize the following: First, increasing initial particle concentration reduces the time to shell formation $\left(t^{*}\right)$ decreases, resulting in larger sizes of the shell. On the other hand smaller initial concentrations allow more time for particle to redistribute themselves and lead to solid "microcrystal" [18] at the end of evaporation. Second, with smaller liquid-solid contact angles $(\theta)$, the effective surface area for evaporation is larger as the particle is more immersed in the liquid; smaller $\theta$ would thus allow evaporation to follow more closely the $d^{2}$-law. Third, particle mobility within the liquid would also affect their redistribution and thus the time to shell formation; high mobility allows particle to escape from early inclusion and thus longer $t^{*}$. Fourth, an increase in evaporation rate $(K)$ should also decrease the time to shell formation, favoring hollow shell formation. Factors of the third and the fourth hypothesis 
can be combined into a non-dimensional Peclet number $(\mathrm{Pe})$, the ratio of the evaporation and particle diffusion rate.

Two recent studies [20, 21] investigated the evaporation characteristics of evaporation of nanofluid droplets containing various insoluble nanoparticles and several hydrocarbon liquids, respectively. It was found (a) that the value of $K$ does not remain constant even before shell formation, (b) that $K$ decreases with time and with decreasing droplet diameter, and (c) that the deviation from the $d^{2}$-law is more pronounced with higher initial particle concentrations. The authors of [20] thought the variation in $K$ to be due to increases in an apparent enthalpy of vaporization with no clear physical explanation. In [21] it attributed the decreased evaporation rate to the particle aggregates within the droplet blocking the liquid pathway to the droplet surface. This study suggests that the deviation results from the particle aggregation inside the droplet, although such blocking is physically unlikely by aggregates residing inside the droplet. A more likely explanation should resemble the surface blockage by particle inclusion at the droplet surface described by Derkachov et al. [18]. Finding (c) appears to agree with the fact that higher initial concentrations should lead to earlier occurrence of inclusion and surface blockage, providing further support for explanation based on surface blockage.

One can find in literature models addressing various aspects of drying and evaporation of droplets containing particles having different sizes, not necessarily at nano-scales (see, for example [22-30]). This paper presents an analytical model for evaporation of droplets containing dilute particle suspension prior to shell formation, based on above findings (a) through (c) and surface blockage [16, 18, 20, 21]. It is expected to address the hypothesis discussed above, with the following specific results: (i) that the model is capable of predicting critical phenomena such as the diameter $\left(d^{*}\right)$ and the time at end of the first stage drying $\left(t^{*}\right)$ and, reduction in the $K$ value (i.e. departure from the $d^{2}$-law and how early it might occur during evaporation), and (ii) that these parameters can be explained based on the relative rates of liquid evaporation rate $(K)$, particle size/liquid pari (particle mobility, $D_{p l}$ ) and their ratio, Peclet number (defined as $P e \equiv K / 8 D_{p l}$ ) [31], the initial particle concentration $\left(Y_{\mathrm{vo}}\right)$ and the contact angle $(\theta)$. Evaporation of sessile droplet is not within the scope of this study (some results can be found in [32-34]). 


\section{The Analytical Model}

Consider a single droplet containing nano-sized insoluble spherical particles that are initially evenly distributed within the droplet, as shown in Fig. 1 (phase (i)). As the liquid evaporates, some particles from the evaporated volume are "trapped" at the droplet surface ("inclusion" as described in [18] and phase (ii) in Fig. 1). These included particles are partly immersed in the liquid [11, 35, 36] and the exposed area of these included particles represent a reduction in the droplet surface from which liquid evaporate. The reduced (and effective) surface area for evaporation is denoted as $A_{e}$ in Fig. 1, while the nominal surface of the droplet is $A_{t}$ (the total area). Further evaporation and reduction of the droplet diameter leads to more included particles on the droplet surface. When the surface concentration of the particles reaches its saturated value at the droplet surface, a shell of densely packed particles forms at its surface [17] (phase (iii) in Fig. 1).
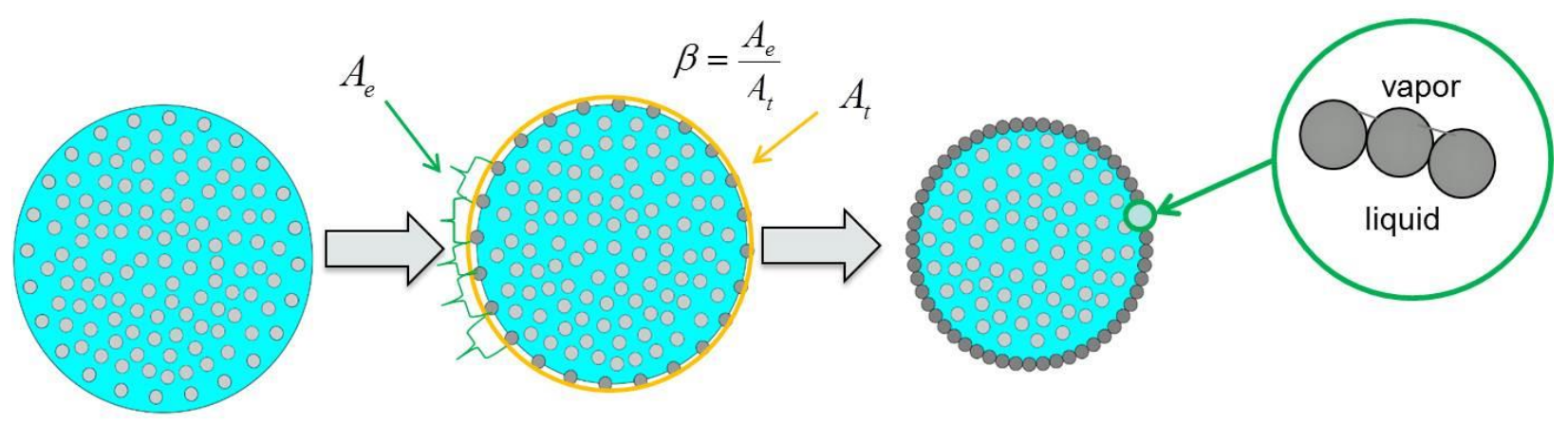

Figure 1 - Evaporation of liquid suspension droplet

Therefore the shell is a layer of densely packed partially wetted particles. Since the particle size is far smaller than the liquid droplet size of engineering interest, the effect of curvature in the liquid-particle interfacial region is negligible [37]. The total of the interstitial area is thus $A_{e}$, which depends on the wettability and the contact angle of the given liquid-particle pair [37]. 


\subsection{Effect of Peclet number on shell size}

The particle motion depends on its diffusivity in the liquid, $D_{p l}$, which is calculated from Einstein-Stokes relation: $D_{p l}=k_{B} T / 6 \pi \eta r_{p}$, where $k_{B}, T, \eta$, and $r_{p}$ are Boltzmann constant, temperature, liquid viscosity, and the particle radius, respectively. The relative motion of the regressing surface and the particles can be characterized by a dimensionless parameter, Peclet number $(\mathrm{Pe})[17,38]$. The characteristic time required for particles to diffuse across the droplet radius $\left(\tau_{d}=(d / 2)^{2} / D_{p l}\right)$ and the lifetime of a droplet is $\tau_{e}=d^{2} / K$, where $d$ is the diameter of the droplet. Therefore,

$$
P e \equiv K / 8 D_{p l}
$$

The scenarios for the extreme cases of $P e \ll 1$ and $P e \gg 1$ are shown in Fig. 2. For $P e \ll 1$, the evaporation rate of a droplet is sufficiently low so that particles have sufficient time to redistribute themselves by diffusion throughout the droplet, resulting in a packed spherical particle aggregate. In this case, the size of final particle aggregate is determined by the initial number of particles, as would be for the microcrystal described in [18]. For $P e \gg 1$, particles have insufficient time to diffuse and are quickly included on the regressing droplet surface, resulting in a hollow or doughnut grains $[39,40]$. For finite value of $P e$, the shell diameter $\left(d_{s}\right)$ depends on the value of $P e$ and the initial particle concentration $\left(C_{o}\right)$, as expected. The combination of $P e$ and $C_{o}$ can be expected to affect the time to shell formation $\left(t^{*}\right)$ and the diameter of the shell $\left(d^{*}\right)$. If the environment is heated, as is the case in drying and combustion applications, the value of $P e$ is significantly larger than 1 . 


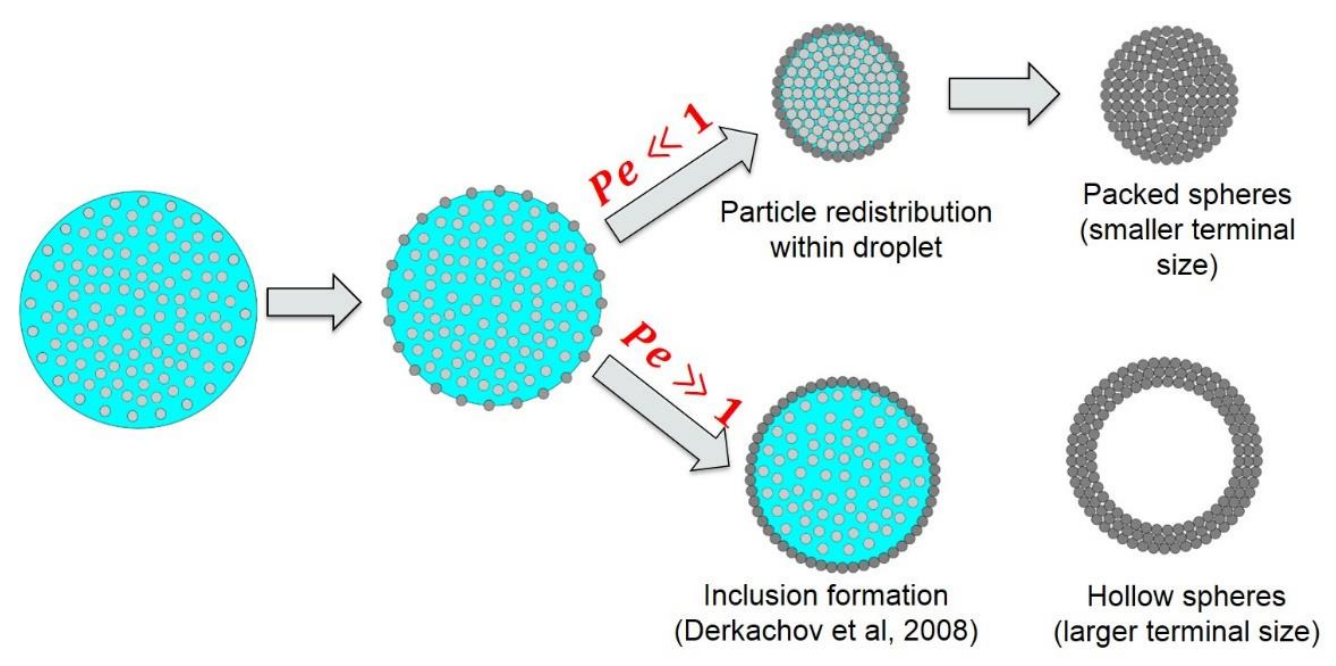

Figure 2 - Effect of particles on the morphology of dry particles

\subsection{Effects of particle inclusion on droplet surface}

For quasi-steady evaporation, the conservation of mass in the gas phase can be written as [9]

$$
4 \pi r^{2}(\rho v)\left(\frac{d Y}{d r}\right)=\frac{d}{d r}\left[4 \pi r^{2} \rho D\left(\frac{d Y}{d r}\right)\right]
$$

where $v, Y$, and $D$ are, respectively, the gas velocity, the mass fraction of liquid vapor, and the mass diffusion coefficient of the liquid vapor. Applying the boundary conditions at the surface [9], Eqn. (2) becomes

$$
\rho_{l} \mathrm{~A}_{t} \dot{r}_{s}=\rho_{s} A_{e} v_{s}=\rho_{s} Y_{s} v_{s} A_{e}-\rho D\left(\frac{d Y}{d r}\right)_{s} A_{e}
$$

where $\rho_{l}$ is the liquid droplet density and $\dot{r}_{s}$ the rate of change of droplet radius, and the subscript $s$ denotes conditions at the droplet surface. For the pure liquid droplet $A_{\mathrm{t}}=A_{\mathrm{t}}$, as shown in Fig.1. Equation (3) states that the bulk mass flow rate $\left(\rho_{s} A_{e} v_{s}\right)$ leaving the droplet surface is equal to the amount of the liquid vapor that is being evaporated plus the amount of the liquid vapor that diffuses back to the surface. In Eqn. (3), $D \rho$ is relatively independent of temperature and pressure so that $D \rho=D_{\mathrm{s}} \rho_{\mathrm{s}}$ [9]. It is also known that the vapor velocity leaving from the liquid surface, $v_{\mathrm{s}}$, is related the surface vapor mass fraction by the following expression: 


$$
v_{\mathrm{S}}=\frac{D\left(\frac{d Y}{d r}\right)_{\mathrm{S}}}{Y_{S}-1}
$$

Substituting Eqn. (4) into Eqn. (3) yields

$$
v_{s}=-\beta \frac{\rho_{1}}{\rho_{\mathrm{s}}} \frac{d r_{\mathrm{s}}}{d t}
$$

where $\beta=A_{\mathrm{e}} / \mathrm{A}_{\mathrm{t}}$. Solving Eqn. (4) and incorporating surface conditions leads to

$$
\frac{r_{s} v_{S}}{D_{\mathrm{S}}}=\ln \frac{Y_{\infty}-1}{Y_{S}-1}
$$

Combining Eqns. (5) and (6) leads to the governing equation for the droplet diameter as a function of time:

$$
\frac{d d^{2}}{d t}=-K^{\prime}=-\frac{8 D_{s} \rho_{S}}{\rho_{l}} \ln \frac{Y_{\infty}-1}{Y_{S}-1}
$$

where $K^{\prime}$ is the effective evaporation rate constant. Comparing the pure liquid result for $K$ [35], one finds

$$
\beta=K^{\prime} / K=A_{e} / \mathrm{A}_{t}
$$

Equation (8) indicates that the droplet evaporation rate gradually decreases as the number of particles on the surface increase before the shell forms. For $\beta$, the rate of particle inclusion on the droplet surface, and thus $A_{e}$, has to be determined.

The partially submerged particle on the droplet surface is shown in the schematic in Fig. 3. It can be seen that $A_{e}=\mathrm{A}_{t}-A_{p}$, where $A_{p}$ is the droplet surface area occupied by the particles at the surface. The area $A_{p}$ is the product of area that occupied by a single particle $\left(A_{s p}\right)$ and the surface particles number $\left(N_{S}\right)$. Therefore, 


$$
A_{p}=N_{S} A_{s p}
$$

With the nomenclature shown in Fig. 3, $A_{s p}$ is expressed as

$$
A_{s p}=\pi r^{2}=\pi\left(r_{p} \sin \phi\right)^{2}
$$

Geometric consideration of the angles shown in Fig. 3 leads to

$$
\phi=\theta+\varphi
$$

where $\theta$ is the contact angle of the particle on the liquid-gas interface. As previously noted, the deformation of the liquid surface at the contact region of particles can be neglected and, consequently, the liquid surface can be assumed to be flat [37], resulting in $\varphi=0$. Therefore

$$
\phi=\theta
$$

Consequently Eqn. (10) becomes

$$
A_{s p}=\pi r^{2}=\pi\left(r_{p} \sin \theta\right)^{2}
$$




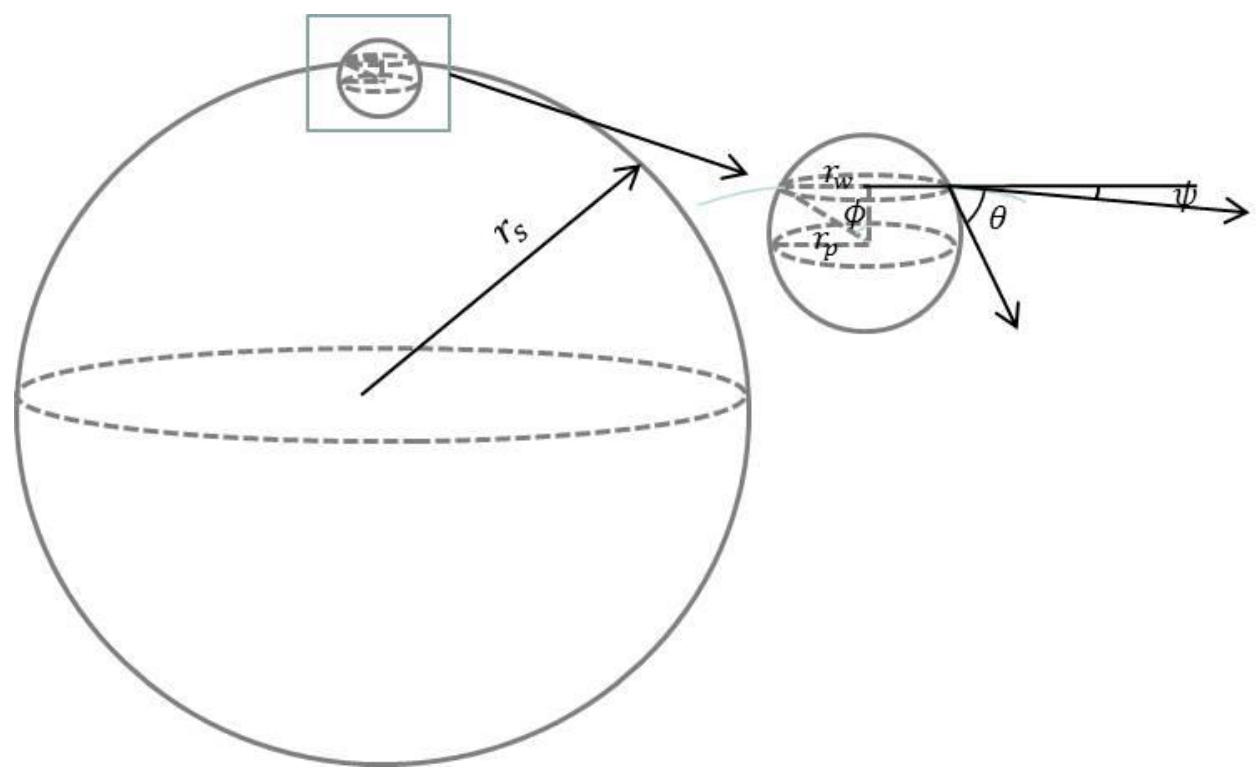

Figure 3 - Parameters related to a single particle trapped at the liquid-particle-gas interface

It is now desirable to determine $N_{s}$ in Eqn. (9) as a function of time. As droplet surface regresses, the particle concentration on and near the surface increases, while the concentration near the droplet center takes a longer time to adjust to the regression, resulting in a concentration gradient in the radial direction. To obtain the instant surface particle number density $\left(C_{s}\right)$, Fick's second law of diffusion is adopted, following [40], to account for the two competing process involved in the evaporation process - droplet receding and particles diffusion:

$$
\frac{\partial C}{\partial t}=\frac{D_{p l}}{r_{s}^{2}}\left(\frac{\partial^{2} C}{\partial R^{2}}+\frac{2 \partial C}{R \partial R}\right)+\frac{R \partial C \partial r_{S}}{r_{S} \partial R \partial t}
$$

where $C$ is the instantaneous number density of particles as a function of time and location, $D_{p l}$, $r_{s}$, the droplet radius, and $R$, the normalized radial coordinate, $R=r / r_{s}$. The analytical solution of Eqn. (14) is provided in [40, 41] and it is 


$$
C_{s}=C_{m} \frac{\exp \left(\frac{K}{8 D p l} \cdot R^{2} / 2\right)}{3 \int_{0}^{1} R^{2} \exp \left(\frac{K}{8 D p l} \cdot R^{2} / 2\right) d R}
$$

where $C_{m}$ is the average particle number density throughout the entire droplet and is described as, with $d_{0}$ being the initial droplet diameter,

$$
C_{m}=C_{0}\left(\frac{d_{0}}{d}\right)^{3}
$$

The combined results of Eqns. (15) and (16) suggest that both $C_{s}$ and $C_{m}$ are functions of $C_{0}$ and $d_{0}$, the initial particle density and initial droplet diameter, respectively. Recalling $P e \equiv$ $K / 8 D_{p l}$, these two equation can be combined to yield the following:

$$
C_{s}=C_{m} \frac{\exp \left(P e \cdot R^{2} / 2\right)}{3 \int_{0}^{1} R^{2} \exp \left(P e \cdot R^{2} / 2\right) d R}=C_{o}\left(\frac{d_{o}}{d}\right)^{3} \frac{\exp \left(P e \cdot R^{2} / 2\right)}{3 \int_{0}^{1} R^{2} \exp \left(P e \cdot R^{2} / 2\right) d R}
$$

With $C_{S}$ known, $N_{s}$ can be obtained for a given $r_{p}$ by accounting the particles number in the control volume where the first layer particles reside. Let the thickness of the first layer particles be $\delta$. Then $\delta=d_{p}$ is a good approximation. Therefore,

$$
N_{s}=C_{s}\left(\pi d^{2} \delta\right)=\pi C_{s} d^{2} \delta
$$

By incorporating Eqns. (8), (9), (13), and (18), one finds

$$
\beta=\frac{A_{t}-A_{p}}{A_{t}}=1-\frac{N_{s} A_{s p}}{A_{t}}=1-C_{s} \alpha\left(\frac{\pi d_{p}^{3}}{4}\right)=1-\frac{\alpha d_{p}^{3}}{4} C_{o}\left(\frac{d_{o}}{d}\right)^{3} \frac{\exp \left(P e \cdot R^{2} / 2\right)}{3 \int_{0}^{1} R^{2} \exp \left(P e \cdot R^{2} / 2\right) d R}
$$

where $\alpha=\sin ^{2} \theta$ and $d_{p}$ is the particle diameter. Combining Eqns. (7) and (18) leads to

$$
K^{\prime}=\beta K=K\left(1-\frac{\alpha d_{p}^{3}}{4} C_{o}\left(\frac{d_{o}}{d}\right)^{3} \frac{\exp \left(P e \cdot R^{2} / 2\right)}{3 \int_{0}^{1} R^{2} \exp \left(P e \cdot R^{2} / 2\right) d R}\right)
$$


where

$$
E=\frac{\exp \left(P e \cdot R^{2} / 2\right)}{3 \int_{0}^{1} R^{2} \exp \left(P e \cdot R^{2} / 2\right) d R}
$$

In terms of particle volume fraction on the droplet surface, $Y_{\mathrm{v}}$ (replacing $C_{o}$ ), Eqn. (20) can be rewritten as

$$
\beta=1-\frac{3}{2 \pi} \alpha Y_{\mathrm{vo}} E\left(\frac{d_{o}}{d}\right)^{3}
$$

The applicability of Eqn. (22) is conditioned upon a sufficient number of particles that constitute a sufficient volume fraction of the droplet. For a single big particle with the same initial volume fraction $Y_{\mathrm{vo}}$, this equation is not valid. For nanoparticles, the number of particles should be sufficient. The results of Eqns. (19) and (20) suggest that the effective surface area and the evaporation both decrease with $C_{s}$ and $d_{p}$, as expected. Equation (16) indicates that the value of $C_{s}$ depends on $P e$ and $C_{m}$, with the latter being a function of time. Equation (20) also suggests that $E$ is a function of $P e$. The $E$ vs. $P e$ relationship is shown in Fig. 4. 


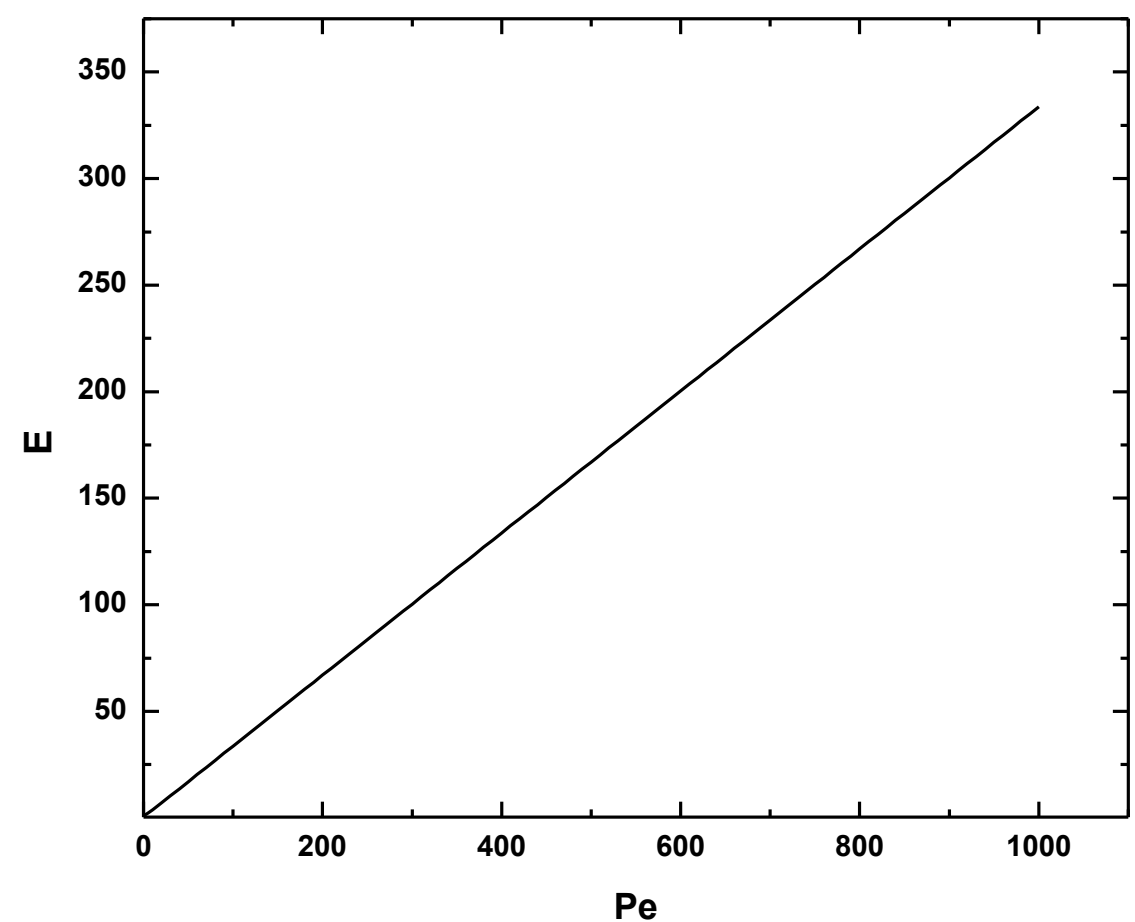

Figure $4 E$ as a function of $P e$.

The parameter $\alpha$ depends on the contact angle, $\theta$, the liquid-particle pair. To the authors' knowledge, no contact angle has been determined on a solid surface with nano-scales. Determining values of $\theta$ is a challenge due to the surface treatment of the particle, as well as limitations for the particle size that can be observed under the optical microscope [36]. In reality $\theta>0$ during the evaporation and the included particles are partially immersed in the liquid (Fig. 3 ). For colloidal stability (with or without the aid of surfactant), $0<\theta<\pi / 2$ is expected and the true angle is more likely to be on the smaller side of this range. During the drying process, the value of $\theta$ is not constant, much like during receding process of a sessile droplet. The value of $\theta$ of the same liquid droplet on a plate differ from that on a nano-sized particle due to its very large curvature [36]. As a reference, the contact angle of 3.9 micrometer-sized polystyrene particles at the air-water interface was measured to be $73^{\circ}[35]$. 
Contact angle data for nano aluminum particles at air-ethanol interface and glass beads and $\mathrm{Fe}_{2} \mathrm{O}_{3}$ particles at air-water interface are rare. It is known that to suspend particles in the liquid, the wettability should be adjusted to make the particles hydrophilic $\left(\theta<90^{\circ}\right)$, which is the reason that investigators used surfactants to suspend the particles [20,21]. In this study, the contact angle is assumed to be constant throughout the evaporation process and is $\theta=40^{\circ}$ is used (along with values for other solid-liquid pairs, in Table 1). The effect of choosing other values of $\theta$ will be discussed following the modeling results using $\theta=40^{\circ}$ and $73^{\circ}$.

The solution for $\beta$ and $K^{\prime}$ involves solving the above equations for $d, C_{m}$, and $C_{s}$ as functions of time. The initial step assumes that $K^{\prime}=K$ (i.e. $C_{s}=0$ ). At the next time step, new values of $\beta$ and $K^{\prime}$ can be calculated and the calculations continues forth in a similar manner until the shell forms.

\section{Results and discussion}

\subsection{Effects of Peclet Number $(P e)$}

To evaluate the performance of the model, recently available experimental data of evaporation of liquid droplets in Refs. [16, 18, 20,21] are used. The liquid and particle properties, StokesEinstein diffusivities $\left(D_{p l}\right)$, and Peclet numbers $(P e)$ used for the calculation are provided in Table 1. Because the evaporation rate of liquid differs depending on experimental conditions (such as the temperature and humidity of the surrounding gas), the value of $K$ is determined by re-plotting the size history plots in these experiments in the form of $d^{2}=d(t)$ and the time derivative of $d^{2}$ at $t=0$ is $K$. The $K$ value thus determined is then used for $\beta=K^{\prime} / K$.

In the model the termination of first drying stage (at $t=t^{*}$ ) is defined as when a dense packed particles forms a shell with the maximum compactness. The maximum compactness is associated with the maximum theoretical solid volume fraction at the surface of the regressing droplet for orthorhombic package of uniform size hard spheres [35]. The commonly used value of this maximum surface volume fraction (denoted by $Y_{\mathrm{vs}}{ }^{*}$ ) ranges approximately from 0.6 to 0.65 . The 
current study adopts $Y_{\mathrm{vs}}{ }^{*}=0.6$ of Minoshima et al. [28]. It is noted that there exists a $C_{\mathrm{s}}{ }^{*}$ corresponding to $Y_{\mathrm{vs}}{ }^{*}$, with a conversion factor depending on the particle size, to be discussed later with the model results.

Figure 5 shows the comparison between experimental data from [18] and the model. The experimental setup allows levitation by electric dynamic balance (EDB) of water droplets having an initial diameter of $d_{o}=26 \mu \mathrm{m}$ and containing $0.1 \%$ (by volume) nano-polysterene particles. The present model appears to predict reasonably well the time history of $d^{2} / d_{o}^{2}$ during the first drying stage, using $P e=3.2$ calculated fromthe experimental conditions. The first stage ends when the value of $d^{2} / d_{o}^{2}$ approaches a constant at $t / d_{o}^{2} \approx 1,700 \mathrm{~s} / \mathrm{mm}^{2}$, attributed to the formation of a shell whose diameter does not change further. 
Table 1. Parameters used for the validation of analytical model, $D_{p l}, K$, and $P e$ calculated based on experimental conditions

\begin{tabular}{|c|c|c|c|c|c|c|c|c|c|}
\hline Refs. & $\begin{array}{l}\text { Expt'l } \\
\text { Conditions }\end{array}$ & Base Fluid & $\begin{array}{l}\text { Initial Droplet } \\
\text { size }\end{array}$ & $\begin{array}{l}\text { Particles } \\
\text { and size }\end{array}$ & $\begin{array}{l}\text { Concentration } \\
\left(1 / \mathrm{m}^{3}\right)\end{array}$ & $\begin{array}{l}\text { Contact } \\
\text { angle } \theta\left(^{\circ}\right)\end{array}$ & $\begin{array}{l}D_{p l} \\
\left(\mathrm{~m}^{2} / \mathrm{s}\right)\end{array}$ & $\begin{array}{l}K \\
\left(\mathrm{~m}^{2} / \mathrm{s}\right)\end{array}$ & $\begin{array}{l}\text { Peclet No. } \\
(P e)\end{array}$ \\
\hline [16] & $\begin{array}{l}\text { Acoustic } \\
\text { Levitation } \\
@ 288 \mathrm{~K}\end{array}$ & Water & $1.5 \mathrm{~mm}$ & $\begin{array}{l}\text { Silica, } \\
200 \mathrm{~nm}\end{array}$ & $4.58 \times 10^{17}$ & 40 & $2.22 \times 10^{-12}$ & $1.09 \times 10^{-9}$ & 61.9 \\
\hline [18] & $\begin{array}{l}\text { Electrical } \\
\text { Dynamic } \\
\text { Balance }\end{array}$ & Water & $1.5 \mathrm{~mm}$ & $\begin{array}{l}\text { Polysterene, } \\
200 \mathrm{~nm}\end{array}$ & $2.39 \times 10^{17}$ & 73 & $2.20 \times 10^{-12}$ & $5.65 \times 10^{-11}$ & 3.2 \\
\hline \multirow[t]{4}{*}{ [21] } & $\begin{array}{l}\text { Pendant } \\
\text { Nat. Conv. } \\
@ 300 \mathrm{~K}\end{array}$ & Ethanol & $1.0 \mathrm{~mm}$ & $\mathrm{Al}, 80 \mathrm{~nm}$ & $2.76 \times 10^{19}$ & 40 & $5.49 \times 10^{-12}$ & $3.0 \times 10^{-9}$ & 68 \\
\hline & $\begin{array}{l}\text { Pendant } \\
\text { Weak } \\
\text { Conv. } \\
@ 300 \mathrm{~K}\end{array}$ & Ethanol & $1.0 \mathrm{~mm}$ & $\mathrm{Al}, 80 \mathrm{~nm}$ & $2.76 \times 10^{19}$ & 40 & $5.49 \times 10^{-12}$ & $9.5 \times 10^{-9}$ & 216 \\
\hline & $\begin{array}{l}\text { Pendant } \\
\text { Weak } \\
\text { Conv. } \\
\text { @ 330K }\end{array}$ & Ethanol & $1.0 \mathrm{~mm}$ & $\mathrm{Al}, 80 \mathrm{~nm}$ & $2.76 \times 10^{19}$ & 40 & $9.49 \times 10^{-12}$ & $1.62 \times 10^{-8}$ & 216 \\
\hline & $\begin{array}{l}\text { Pendant } \\
\text { Weak } \\
\text { Conv. } \\
\text { @ } 380 \mathrm{~K}\end{array}$ & Ethanol & $1.0 \mathrm{~mm}$ & $\mathrm{Al}, 80 \mathrm{~nm}$ & $2.76 \times 10^{19}$ & 40 & $2.32 \times 10^{-11}$ & $3.52 \times 10^{-8}$ & 246 \\
\hline
\end{tabular}




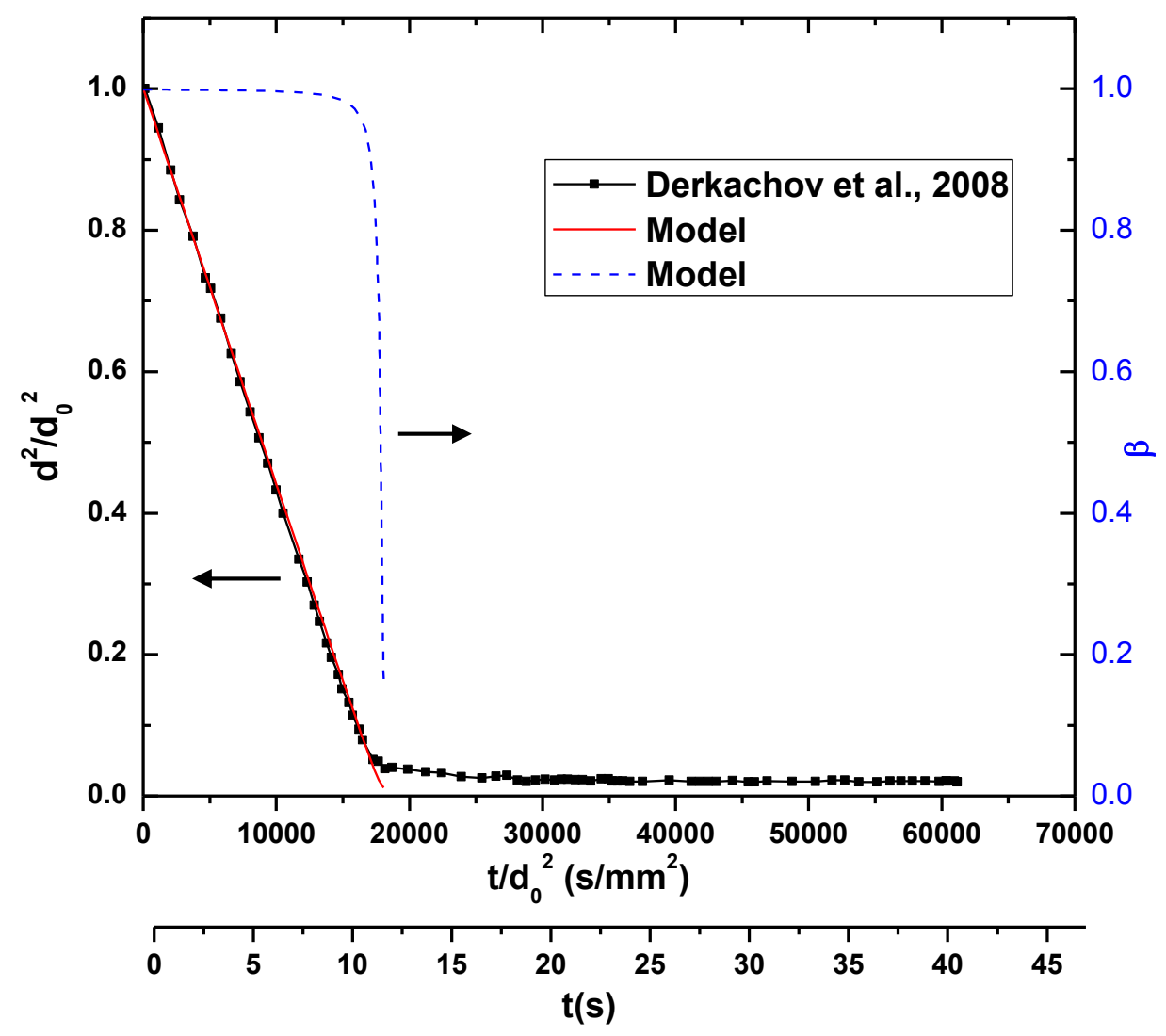

Figure 5 Evaporation of water droplet with $0.1 \%$ (by volume) nano-polystyrene particles $(P e=3.2)($ two horizontal axis are needed)

Built upon the success of predicting $d^{2} / d_{o}^{2}$, the value of $\beta$ is then calculated and also presented in Fig. 5. The $d^{2} / d_{o}^{2}$ vs. $t / d_{o}^{2}$ during the first stage drying shown in Fig. 3 appears to be a straight line, suggesting that the evaporation process closely following the $d^{2}-$ law. It is thought that small $P e$ (in this case of order of 1) the particles within the droplet has time allowed by the evaporation rate (i.e., large $D_{p l}$ comparable to $K$ ) to redistribute. Consequently during the first stage, the droplet surface is relatively free of particles or has very few inclusions and the evaporation closely resembles that of a pure liquid droplet. For the same reason, the value of $\beta$ remain relatively constant, near unity, until $t / d_{o}^{2} \approx 1,500 \mathrm{~s} / \mathrm{mm}^{2}$, followed by a precipitous decrease between $t / d_{o}^{2} \approx 1,500 \mathrm{~s} / \mathrm{mm}^{2}$ and $t / d_{o}^{2} \approx 1,700 \mathrm{~s} / \mathrm{mm}^{2}$. The rapid decrease in $\beta$ prior to the shell 
formation can be explained as follows. First, the particle concentration increases according to $d^{-3}$ that rapidly increases with time. Secondly, the droplet surface decreases as $d^{2}$, which, when coupled with the concentration increase, produces an surface concentration $C_{s} \propto d^{-5}$, a nearly catastrophic increase that leads to the precipitous decrease in $\beta$. As will be discussed alone with the results of Fig. 7 later, the tendency for precipitous decreases in $\beta$ is enhanced with decreases in $P e$, as a smaller $P e$ value means that particles have time to redistribute during the evaporation process, leading to particularly higher concentration compare to the case with larger values of $P e$.

Figure 7 shows the good agreements between the model predictions of $d^{2} / d_{o}^{2}$ vs. $t / d_{o}^{2}$ and experimental results of ethanol droplets with $2.5 \%$ nano-Al under natural convection at 300K [21] Several observations can be made of the results of Fig. 7, described in the following.

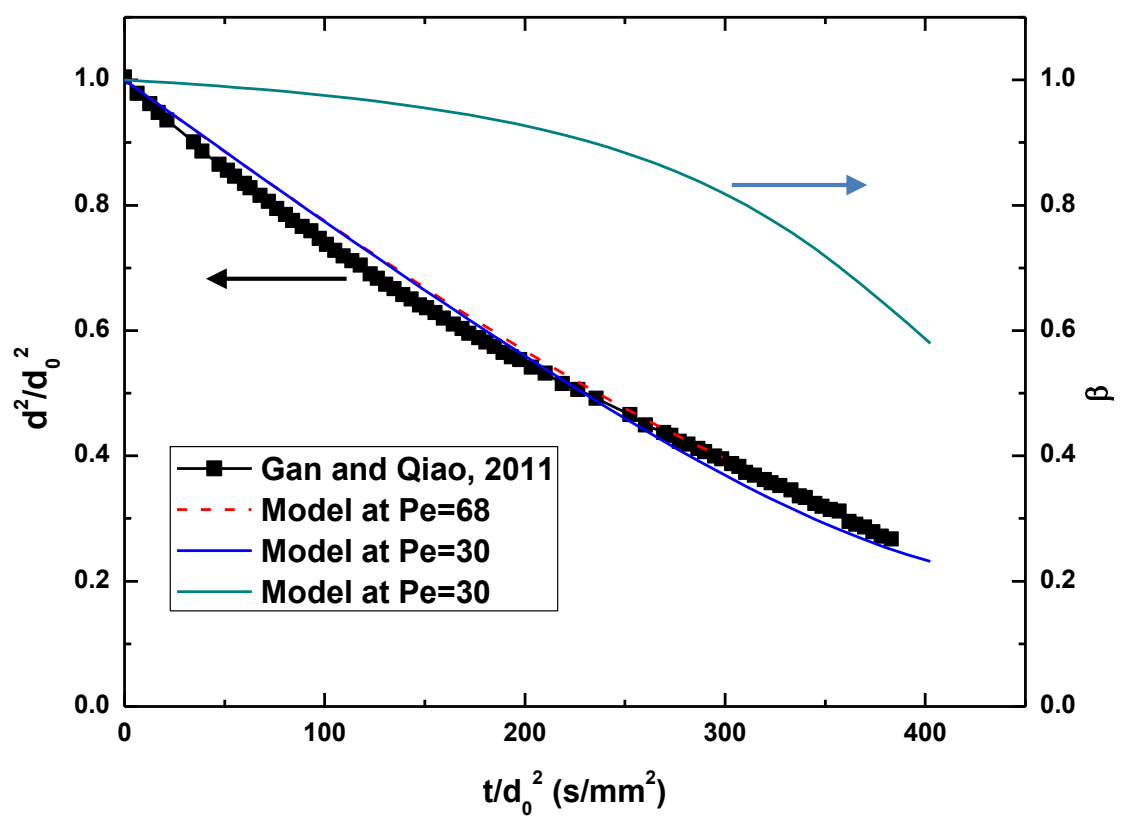

Figure 6 - Evaporation of ethanol droplets containing $2.5 \%$ nano-Al under natural convection at 300K

First, the current model using both $P e=30$ and 68 appears to predict the droplet size history well, with $P e=30$ producing better result for the end of the droplet lifetime and the droplet size 
at that instant, $d^{*}$, i.e., the droplet size at the end of the first drying state. These numerical results is expected because a larger $P e$ indicates a larger rate of inclusion, capturing more particles in the droplet surface, than a smaller $P e$, as already explained by the results in Fig. 2. These results suggests that the real value of $P e$ is somewhere between 30 and 68, likely much closer to the former than the latter, as the result of Fig. 6 would suggest. It is likely that the very weak convection effect in the experiment [21] might cause internal circulation within the droplet and thus increase the effective values of $D_{p l}$.

Secondly, the model predicts, in agreement with the experimental results, a nonlinear $d^{2} / d_{o}^{2}$ vs. $t / d_{o}^{2}$ relationship, suggesting a departure from the pure liquid $d^{2}-$ law. $P e$ in this case (Fig. 6) is an order of magnitude larger than that of Fig. 5. The evaporation rate is relatively large and particle inclusion on the droplet surface at a larger (inclusion) rate. As a result, two events take place: (1) inclusion of particles at the droplet surface happens sooner than that in Fig. 5, and (2) the particle inclusion causes $A_{e}$ to also happen earlier. These events lead to earlier departure from the $d^{2}$-law and earlier decrease in $\beta$ (both in terms of droplet lifetime and in terms of $t / d_{o}^{2}$ compared to Fig. 4), i.e., not so precipitous a decrease near the end of the first stage as seen in Fig. 5. Therefore the disparity of the $P e$ values of Figs. 5 and 6, would further confirm the qualitative results shown in Fig. 2 - that is, a larger $P e$ leads to earlier inclusion and tend to form hollow shells. Furthermore a larger $P e$ also leads to a relative gradual decrease in $\beta$ over a longer time compare to the case with smaller $P e$ values, as already mentioned with the results of Fig. 5, where $P e=3.2$, much smaller than $P e=68$ of Fig. 6 .

Table 2 - Comparison of droplet size at the end of first evaporation stage (Data same as those of [21] shown in Table 1)

\begin{tabular}{|c|c|c|c|}
\hline \multicolumn{2}{|c|}{$\begin{array}{l}\text { Prediction of } d^{*} \text { using } \\
P e \text { based on experiment }\end{array}$} & \multicolumn{2}{|c|}{$\begin{array}{l}\text { Prediction of } P e \text { using } \\
\text { experimental data of } d^{*}\end{array}$} \\
\hline$P e_{\text {exp }}$ & $d_{\text {pre }}^{*}(\mathrm{~mm})$ & $\overline{P e_{\text {pre }}}$ & $d_{\mathrm{exp}}^{*}(\mathrm{~mm})$ \\
\hline 68 & 0.66 & 30 & 0.48 \\
\hline 198 & 0.89 & 25 & 0.46 \\
\hline 216 & 0.94 & 3 & 0.27 \\
\hline 246 & 0.96 & 1 & 0.24 \\
\hline
\end{tabular}


As discussed above, being able to predict $d^{*}$ is one of the indicators (besides predicting the departure from the $d^{2}$-law) of the model's success. Although the effective value of Pe might not be exactly known, Eqn. (17) can be rewritten to express $d^{*}$ as

$$
\frac{d^{*}}{d_{0}}=\left(\frac{C_{0} E}{c_{S}^{*}}\right)^{1 / 3}
$$

where $C_{s}{ }^{*}=0.64$, as discussed earlier. The current model using $P e_{\exp }=68$ (based on experimental conditions) predicts $d_{\text {pre }}^{*}=0.66 \mathrm{~mm}$. A better prediction for the experimental value $d_{\mathrm{exp}}^{*}=0.48 \mathrm{~mm}$ was produced by using the chosen $P e=30$. These values are listed in Table 2, along with Gan and Qiao's result [21] under various conditions. The first set of $P e-d^{*}$ results use $P e_{\text {exp }}$ to produce $d_{\text {pre }}^{*}$, while the second set is to predict best-fitting value of $P e$ (i.e., $P e_{\text {pre }}$ ) based on the experimentally determined $d_{\text {pre }}^{*}$. The first set of results indicate that as $P e_{\exp }$ increases from 68 to $246, d_{\text {pre }}^{*}$ increases from $0.66 \mathrm{~mm}$ to approximately $0.94 \mathrm{~mm}$, which is contrary to the experimental result of $d_{\mathrm{exp}}^{*}$, decreasing from $0.48 \mathrm{~mm}$ to $0.24 \mathrm{~mm}$. A question arises as to whether the experimental value of $P e$ is affected by other effect such as internal circulation within the droplet that enhances the particle mobility. Such a question is reasonable as the experimental conditions in [21] involved weak convection and as $d_{\text {exp }}^{*}$ is experimentally determined.

To answer this question, efforts were made to find the best-fitting value of $P e$ (i.e., $P e_{\text {pre }}$ ) qualitatively consistent with experimental $d^{*}$ (i.e., $d_{\text {exp }}^{*}$ ). The second set of results were produced (also shown in Table 2) that suggest $P e_{\text {pre }}$ decreases with $d_{\text {exp }}^{*}$, consistent with the physical picture depicted in Fig. 2 and the above discussions. 

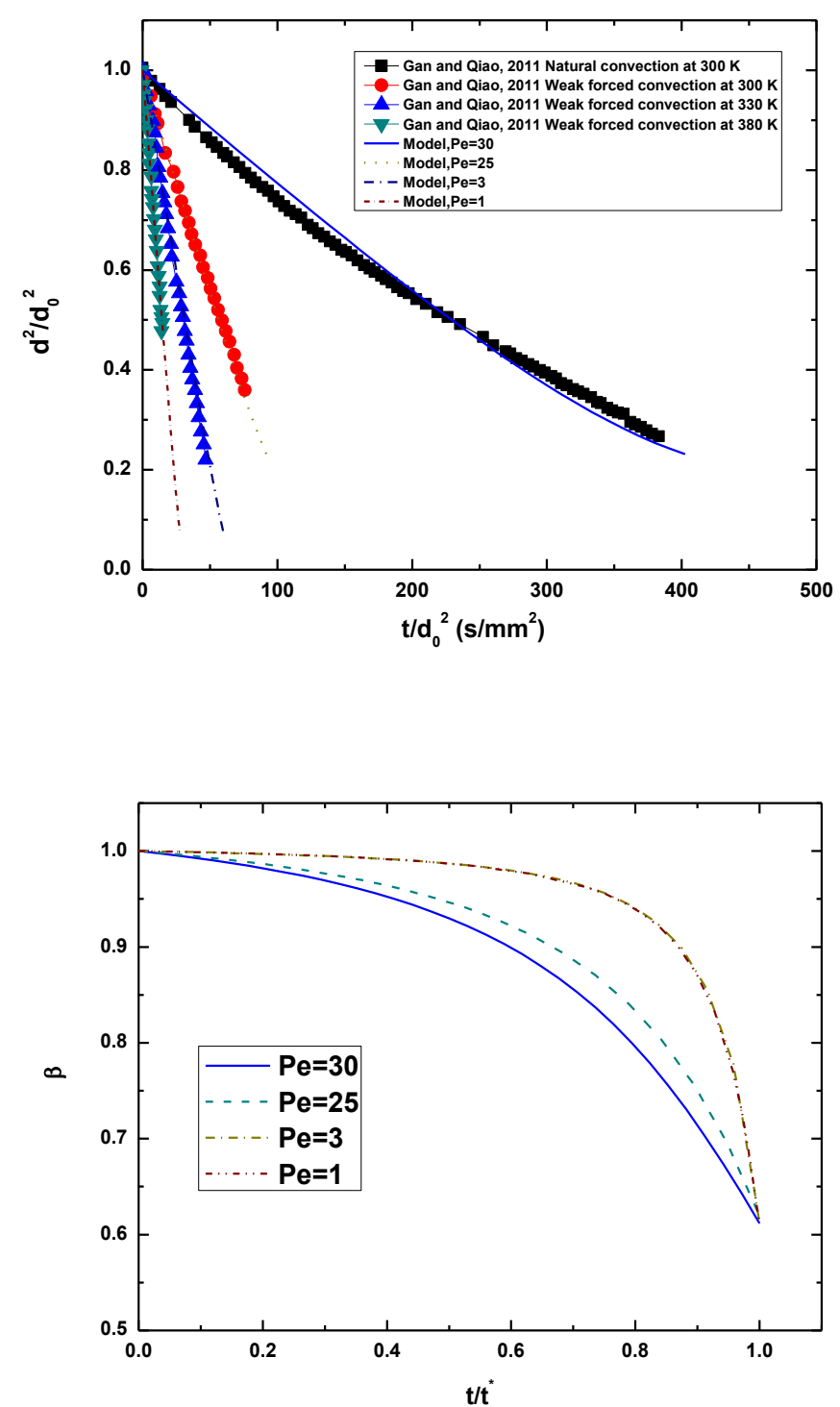

Figure 7 - Droplet size history of ethanol droplets containing 2.5\% nano- aluminum particles under various temperatures [21]

To provide further support for the second set of data, $d^{2} / d_{o}^{2}$ vs. $t / d_{o}^{2}$ data of Table 2 are presented in Fig. 7, using various values of $P e$. It can be seen in Fig. 7 that as $P e$ decreases, the $d^{2} / d_{o}^{2}$ vs. $t / d_{o}^{2}$ curve becomes closer being a straight line, i.e., the $d^{2}$-law is followed more closely throughout the entire first stage of drying/evaporation. Again as $P e$ decreases, the decrease in $\beta$ occurs later toward $t / t^{*}=1$ and the decrease is more precipitous, similar to the findings presented in Figs. 5 and 6. 


\subsection{Further Model Predictions}

Based on the encouraging model predictions of the previous section, this section expands on model predictions that helps to elucidate other parametric effects: the initial particle concentration $\left(Y_{\mathrm{vo}}\right)$ and the contact angle $(\theta)$. It is expected the increasing $Y_{\mathrm{vo}}$ would lead to earlier shell formation and larger shell diameter, similar to the effect of increasing $P e$. Decreasing $\theta$ should delay the decrease in $\beta$ until shell formation, as prior to that time all particles are immersed in the liquid and the evaporation takes place over the nominal surface of the droplet.

\section{Effect of Initial Particle Concentration $\left(Y_{v o}\right)$}

Equation (23) also suggest that $d^{*} / d_{o}$ depends on the initial particle number concentration $\left(C_{o}\right)$ and thus also on $Y_{\text {vo }}$ according to Eqn. (22), besides the $P e$-dependence through $E$. Figure 8 shows the predicted $d^{*} / d_{o}$ as a function of $P e$ for $Y_{\mathrm{vo}}$ (the initial volume fraction of the particles) ranging from 0.01 to 0.1 . $Y_{\text {vo }}$ is used because it is commonly encountered in the literature than the number density $C_{o}$. In the present prediction, aqueous droplet containing spherical aluminum particles of a uniform size of $100 \mathrm{~nm}$ is considered, so according to Eqn. (22) the conversion relationship is $Y_{\text {vo }}=5.24 \times 10^{-22} \times C_{o}$, where $C_{o}$ is in the unit of $1 / \mathrm{m}^{3}$. All the curves in Fig. 8, as in Fig. 7, is determined by the condition $Y_{\mathrm{vs}}{ }^{*}=0.6$. It is noted that, the curves shown in Fig. 8 are those possible solution for the given values of $Y_{\mathrm{vo}}$. It can be seen that there is a limiting combination of $Y_{\mathrm{vo}}$ and $P e$ that determines the end of the first evaporation stage, beyond which the current model is not applicable. Take $Y_{\mathrm{vo}}=0.1$ for example. No result can be obtained for $P e \geq 10$ and for $Y_{\mathrm{vo}}=0.01$ no calculation can be carried out for $P e \geq 185$. In the extreme case of $Y_{\mathrm{vo}} \rightarrow 1$, the condition of $Y_{\mathrm{vs}}{ }^{*}=0.6$ is instantaneously reached when evaporation commences at $t=0$. 


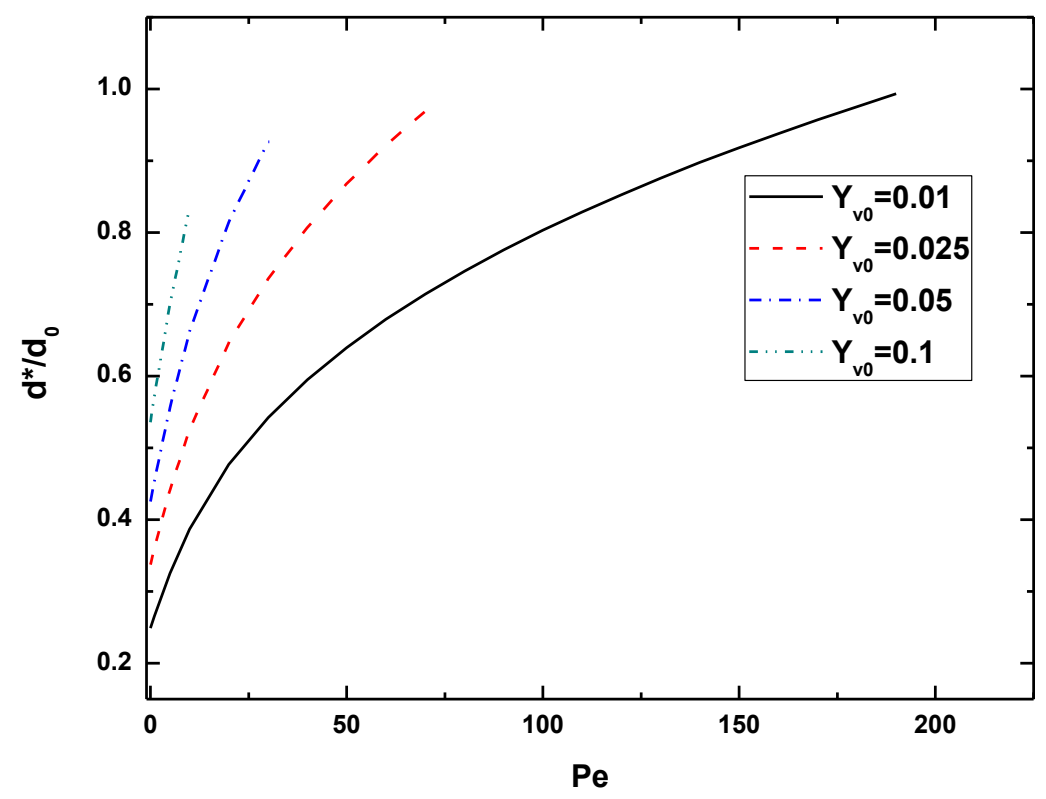

Figure 8 - The droplet size at the end of the first evaporation stage as a function of Peclet numbers under various initial particle volume fractions

The results of Fig. 8 suggest that for a given $Y_{\mathrm{vo}}, d^{*} / d_{o}$ increases with $P e$, in qualitative agreement with the second set of the $P e-d^{*}$ data in Table 2. For a given $P e$, the larger $Y_{\mathrm{vo}}$ is, the earlier $Y_{\mathrm{vs}}{ }^{*}$ is reached earlier producing larger $d^{*} / d_{o}$. These results are consistent with the physical picture described in Fig. 2. Smaller $Y_{\text {vo }}$ values can allow larger $P e$, as fewer particles would be caught on the regressing droplet surface.

The limiting combinations of $Y_{\mathrm{vo}}$ and $P e$ for the first stage of evaporation is further illustrated in Fig. 9 in terms of $t^{*} / t_{\text {life }}$, in addition to the results in terms of $d^{*} / d_{o}$. Here $t_{\text {life }}$ is the droplet lifetime defined as $t_{\text {life }}=d_{o}^{2} / K$. For a given $P e, t^{*} / t_{\text {life }}$ decreases with increasing $Y_{\mathrm{vo}}$, as expected because the particle inclusion rate on the regressing droplet surface increases, thus reaching $Y_{\mathrm{sv}}{ }^{*}$ earlier. On the other hand for a given $Y_{\mathrm{vo}}, t^{*} / t_{\text {life }}$ decreases with increasing $P e$, attaining $Y_{\mathrm{vs}}{ }^{*}$ value earlier. Combining these two reasons, increasing $Y_{\mathrm{vo}}$ beyond a critical value would render $t^{*} / t_{\text {life }} \rightarrow 0$ even for small $P e$-that is, for sufficiently large values of $Y_{\mathrm{vo}}, Y_{\mathrm{vs}}{ }^{*}$ is 
instantaneously reached, just like $d^{*} / d_{o}$ is instantaneously reached when evaporation (or calculation) commences, as described by the results of Fig. 8.

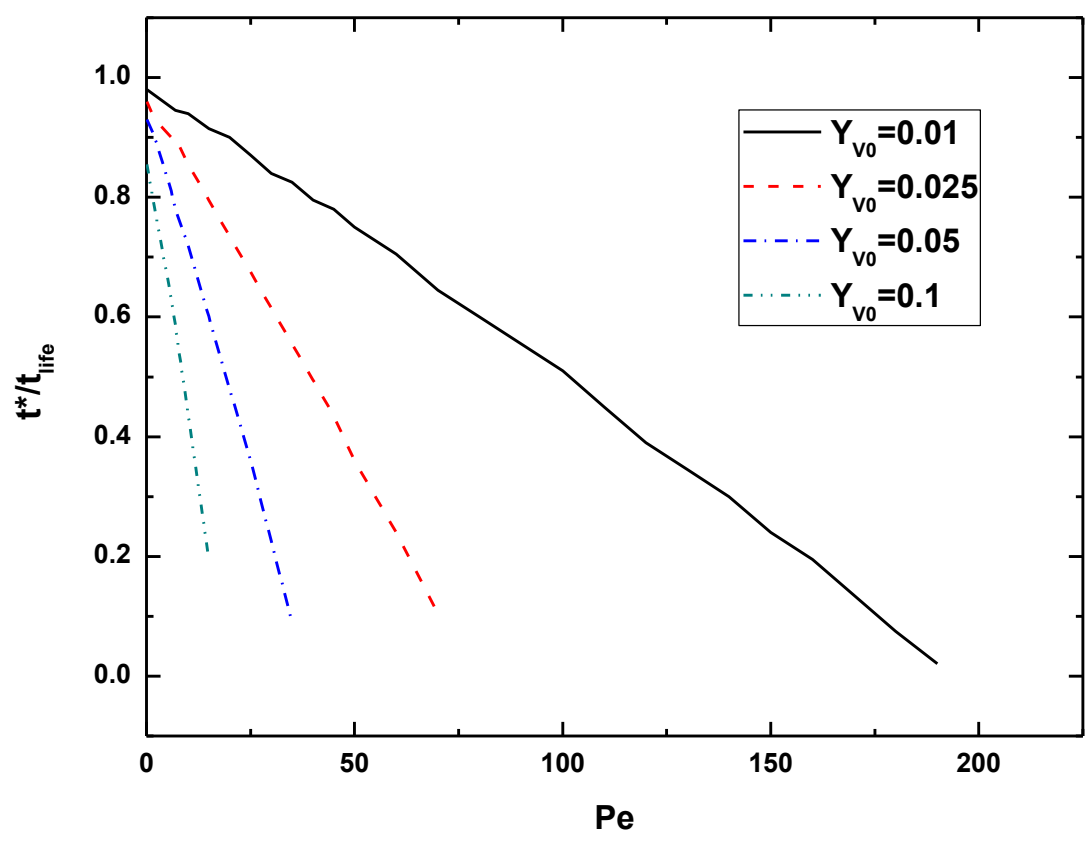

Figure 9 - The duration time of first evaporation (or drying) stage as a function of Peclet numbers under various initial particle volume fractions.

To find the limiting combination of $Y_{\text {vo }}$ and $P e$, Eqn. (23) is re-written as

$$
\frac{d^{*}}{d_{0}}=\left(\frac{Y_{\mathrm{vo}} E}{Y_{\mathrm{vs}}^{*}}\right)^{1 / 3}
$$

Equation (24) provides the criterion for the limiting combinations of $Y_{\mathrm{vo}}$ and $P e$ beyond which the current model ceases to produce results and, in experimental terms, no further evaporation is necessary in order for the shell to form and for the first evaporation stage to end. The result of Figure 4 suggests that

$$
E=\frac{P e}{3}
$$


Therefore, taking $d^{*} / d_{0}=1$ and upon substituting this value and Eqn. (25) into Eqn. (24), the criterion for the first stage drying to be possible is

$$
Y_{\mathrm{vo}} \cdot P e<1.8
$$

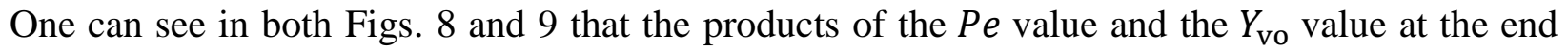
point of each of the four curves yields $Y_{\mathrm{vo}} \cdot P e \approx 1.8$, confirming the limiting condition Eqn. (26). The curve showing the critical initial concentration, $Y_{\mathrm{vo}}$, following $Y_{\mathrm{vo}} \cdot P e=1.8$, is plotted in Fig. 10 , where a steep decrease in $Y_{\text {vo }}$ (the critical initial concentration) with increasing $P e$ is noted for Pe smaller than approximately 20 .

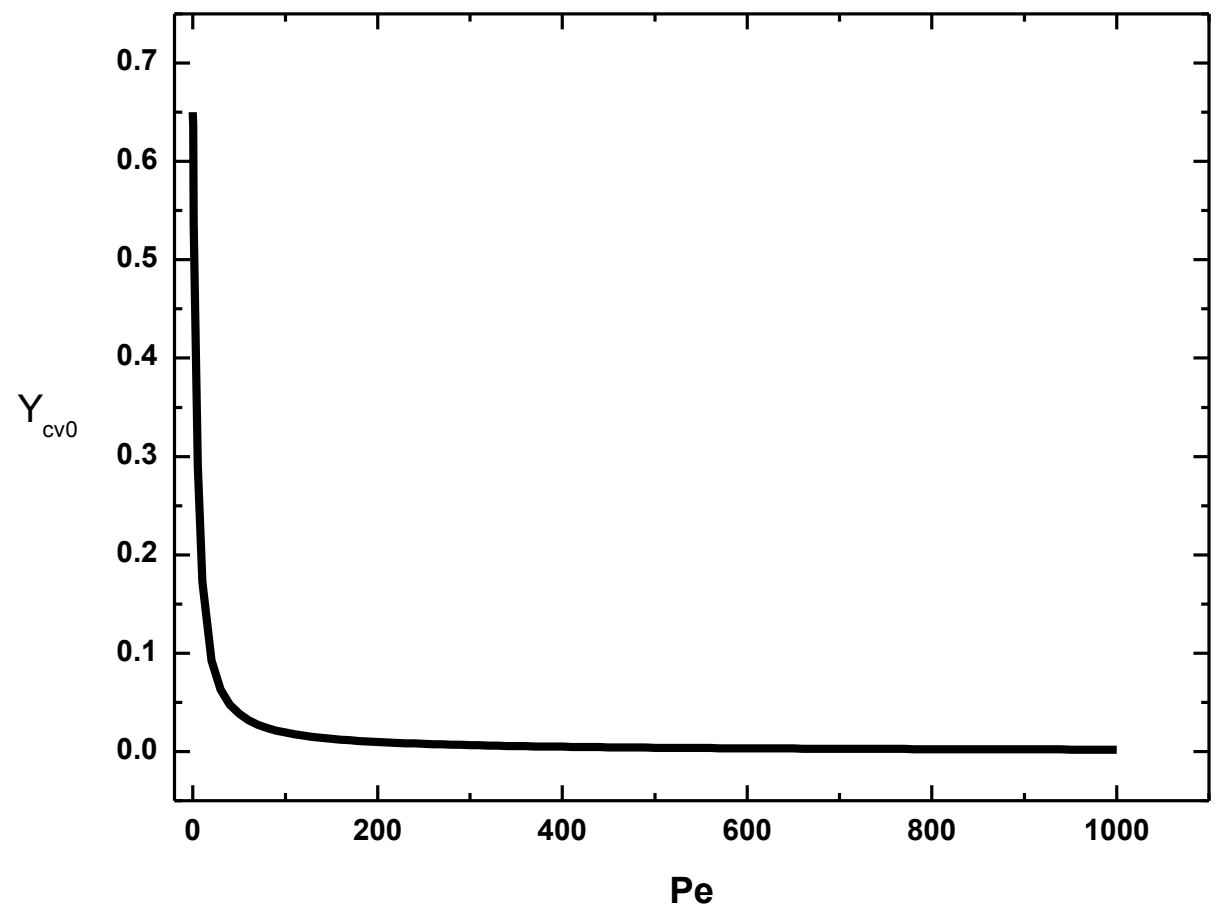

Figure 10 - The criteria for allowing droplet shrinkage in the first evaporation stage 
Therefore to produce large aerodynamic diameters of drug particles for effective delivery, a choice of large values of $P e$ is desirable [31]. When couple with the limiting criterion, a small value of $Y_{\mathrm{vo}}$ has to be chosen. Such requirements lead to $d^{*} / d_{o}-P e$ curves similar to that of $Y_{\mathrm{vo}}=0.01$ in Fig. 8 as opposed to that of $Y_{\mathrm{vo}}=0.1$. In combustion applications, where $P e$ is large, the burning of heavy and slurry fuels tend to produced ceno-spheres with large $d^{*}$ [34]. These two large $P e$ applications produced hollow-sphere results consistent with the depiction of Fig. 2. As the result of Fig. 10 suggests, for combustion applications, hollow spheres and cenospheres are almost always expected. Thus, the large $P e$ and small $Y_{\text {vo }}$ combination leads to early shell formation and, as a result, later and complete evaporation is accomplished when the liquid finds ways to diffuse to the outer surface of the shell, which constitute the second evaporation (or drying) stage. As can be seen from Fig. 9 (for example, $Y_{\mathrm{vo}}=0.01$ ), such a combination is accompanied by small values of $t^{*} / t_{\text {life }}$, i.e., a relatively short first stage and a long second stage. Because of the early shell formation for small $Y_{\mathrm{vo}}$ values, one expects that the sphere thus formed not only has a large aerodynamic diameter but also a small density. There would be a tremendous propensity for such spheres to disperse.

On the contrary, a smaller $P e$ tends to yield large $t^{*} / t_{\text {life }}$ (Fig. 9) and smaller $d^{*} / d_{o}$ (Fig. 8) and the evaporation process spends a shorter time in the second stage. In this case the final particle aggregate is of a more compact form, with less void, than those produced with larger $P e$ and smaller $Y_{\mathrm{vo}}$, as depicted in Fig. 2.

Gerken et al. [42] studied the effect of initial particle concentration in the Al-ethanol nanofluid (particle size range of $40-60 \mathrm{~nm}$ ) droplet evaporation was investigated on its effect on the $d^{2}-$ law. They found the $d^{2}$-law to hold for weight concentration up to $1 \%$ and $d^{2} / d_{o}^{2}<0.75$ and the $K$ value to decrease from $K \approx 0.0063 \mathrm{~mm}^{2} / \mathrm{s}$ for pure liquid to an asymptotic value $K \approx$ $0.0054 \mathrm{~mm}^{2} / \mathrm{s}$ for weight concentration $\geq 1.5 \%$. The qualitative trend in $K$ found in [42] is thus in agreement with those found in [20], but unlike [20] it begins to decrease for concentration slightly greater than zero. These researchers performed a modeling analysis and suggested that the particle packing near the droplet surface is responsible for the decrease in $K$. This group did not address the effect of $P e$ on the lifetime and the shell size at the end of the first stage of evaporation. 


\section{Effect of Contact Angle $(\theta)$}

For the aforementioned results, a realistic contact angle $\theta=40^{\circ}$ has been assumed. The relation $\alpha=\sin ^{2} \theta$ in Eqns. (19) and (20) indicates that $\beta$ depends on the contact angle, $\theta$. For highly wetting liquid $(\theta \rightarrow 0$ and $\alpha \rightarrow 0) \beta \rightarrow 1$ throughout the first drying stage.

The effect of $\theta$ on $d^{2} / d_{o}^{2}$ vs. $t / d_{o}^{2}$ is plotted in Fig. 11 along with the data of acoustically levitated water droplets containing 5 wt. \% nano-silica particles with an ambient temperature of $300 \mathrm{~K}$ [16]. Because acoustic effects greatly enhance liquid circulation [16, 42], making very small $P e$. The value of $D_{p l}$ is enhanced by 1 million times, from the Einstein-Stokes diffusivity of $10^{-12}$ $\mathrm{m}^{2} / \mathrm{s}$ to $10^{-6} \mathrm{~m}^{2} / \mathrm{s}$. Reference [16] adopted the variable liquid-solid diffusion coefficient, which varies with the particle concentration. However, because the intense acoustic wave-driven internal circulation is significant making small values of $P e$. For convenience and especially for dilute suspensions of interest to present model predictions, $D_{p l}$ (and thus $P e$ ) was set to be a constant equal to $10^{-6} \mathrm{~m}^{2} / \mathrm{s}$. Without knowing a priori the value of $P e$, a curve-fitting effort helped to identify $P e=0.05$ to be a good choice for predicting the droplet size history, as shown by the results of Fig. 11 [16], lending support for assuming constant $D_{p l}$ and $P e$. The authors of [16] also presented predictions using the population density (PB) and continuous species transport model (CST) model, also shown in Fig. 11. Although these two model produces satisfactory $d^{2} / d_{o}^{2}$ vs. $t / d_{o}^{2}$ results, no prediction of $\beta$ or the effect of $\theta$ was conducted.

For the small $P e$, both the data and predictions reveal a linear relationship between $d^{2} / d_{o}^{2}$ and $t / d_{o}^{2}$, as expected. The effect of $\theta$ ranging from $10^{\circ}$ to $40^{\circ}$ on the linearity and the quantitative agreement does not appear to be significant, as seen in Fig. 11. The effect of $\theta$ on $\beta$ is more pronounced. Similar to larger values of $P e$ and $Y_{\text {vo }}$, larger values of $\theta$ appear to have similar effect on $\beta$ in that the decreases in $\beta$ begins earlier during the evaporation process. This effect can be attributed to the fact that more particle surface would be exposed leading to a reduction in $A_{\text {eff }}$ and $\beta$. At a given time, for example $t / d_{o}^{2} \approx 800 \mathrm{~s} / \mathrm{mm}^{2}, \beta$ is nearly unity for $\theta=0$ (a perfectly wetting liquid) and is approximately 0.8 for $\theta=40^{\circ}$. One would imagine that for the given $Y_{\mathrm{vo}}$ of the experiment and the same inclusion rate, a smaller $\theta$ means more immersion of the same 
number of included particles in the liquid and thus larger $\beta$ at the same instant of the evaporation process. In the extreme case of $\theta=0$, one can expect no decrease in $\beta$ throughout the entire first stage.

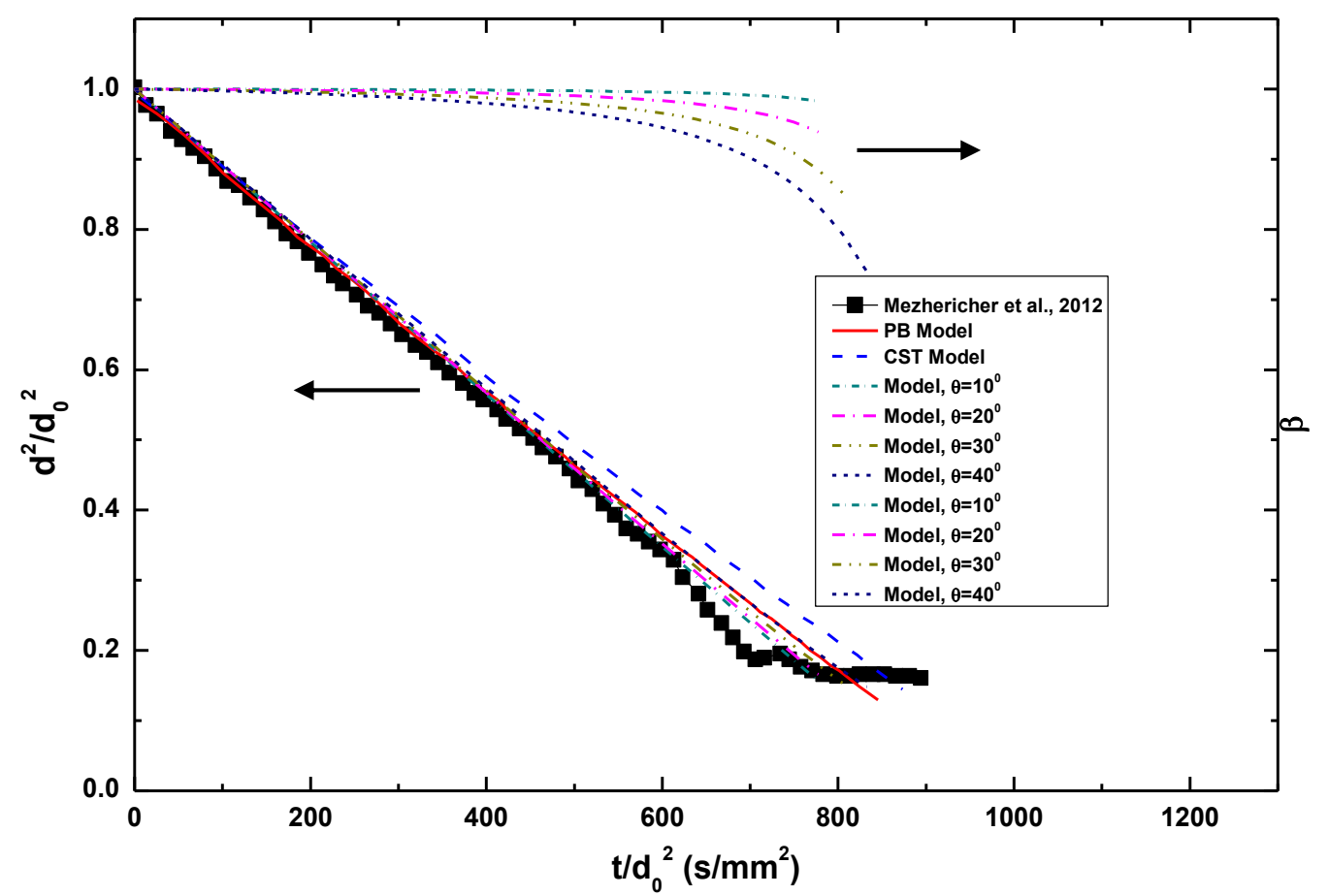

Figure 11 - Comparison between the experiment and various models (acoustic levitated water droplets with 5 wt. \% nano-silica particles [16])

To further examine the analytical reasoning for the above observed effect of $\theta$ on $\beta$, it is decided to examine $\beta^{*}$, the value of $\beta$ at the end of the first stage (i.e., at $Y_{\mathrm{vs}}=Y_{\mathrm{vs}}{ }^{*}$ ). It is noted that $\beta^{*}<\beta$ throughout the first stage, $\beta^{*}$ is a good indicator of the reduction in the evaporation rate constant. To utilize Eqn. (22), set $d=d^{*}$ and choose $E=\pi$ (and $P e=3 \pi$ ) which is a realistic value for which variations of $\beta$ with $t / t^{*}$ are expected, as shown in Fig. 7, and is not too large to necessarily cause $Y_{\mathrm{vo}}$ to be zero (Fig. 10). Corresponding to $d=d^{*}, Y_{\mathrm{vs}}=Y_{\mathrm{vs}}{ }^{*}$ and Eqn. (22) yields

$$
\beta^{*}=1-\frac{3}{2} \alpha Y_{\mathrm{vs}}{ }^{*}=1-\frac{3}{2}\left(\sin ^{2} \theta\right) Y_{\mathrm{vs}}{ }^{*}
$$


The result of Eqn. (27) is plotted in Fig. 12 for the contact angle up to $90^{\circ}$ for the purpose of illustrating the effect of $\theta$, although $\theta=90^{\circ}$ would suggest a non-wetting fluid and unstable particle suspension. It can be seen that $\beta^{*}$ decreases with increased $\theta$. This result arises from the fact that with same number of included particle, the more wetting fluids (smaller $\theta$ ) can "climb" further toward the top of the particle (see Fig. 3), leading to larger $\beta^{*}$.

Equation (27) also suggests that for the same $\theta$, a smaller $P e$ (for example $P e=0.05$ in Fig. 11) produces larger values of $\beta^{*}$ than that by larger Peclet numbers ( $P e=3 \pi$ in Fig. 12). Take $\theta=40^{\circ}$ for example; $P e=0.05$ generates $\beta^{*} \approx 0.75$, while for $P e=3 \pi, \beta^{*} \approx 0.60$. Although such a comparison is made between the predicted results, the results appear reasonable and to be expected. This is because, a larger $P e$ causes a larges particle inclusion rate and thus a smaller $A_{\mathrm{e}}$ and smaller $\beta$ and $\beta^{*}$, as already discussed.

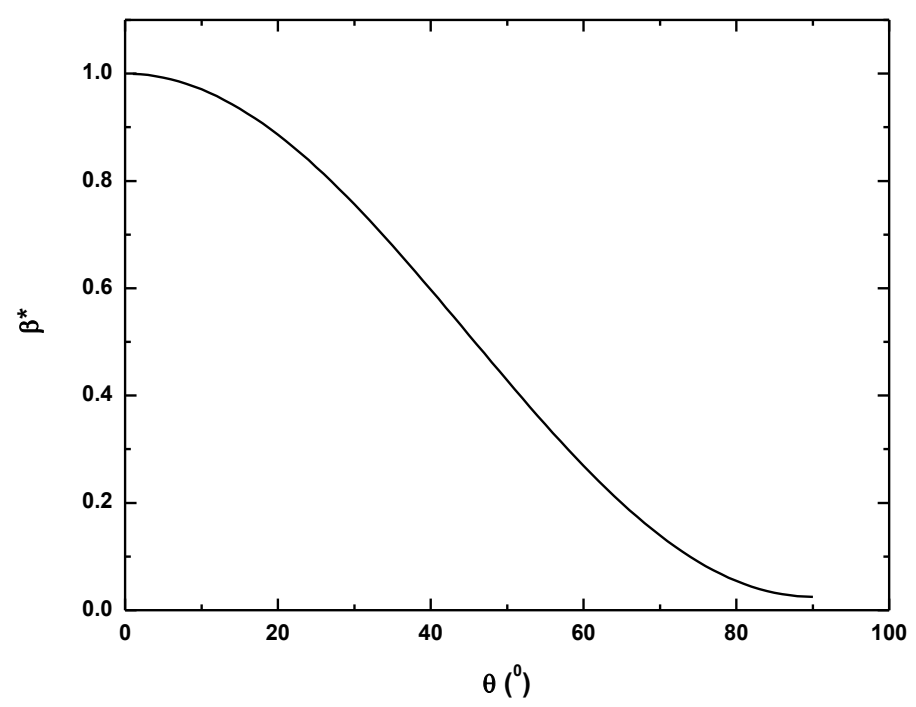

Figure 12 - The evaporation rate ratio at the end of first evaporation stage as a function of contact angle

\section{Conclusion}

The effects of Peclet number $(P e)$, initial particle volume fraction $\left(Y_{\mathrm{vo}}\right)$ and contact angle $(\theta)$ on evaporation of droplets containing particles prior to shell formation can be summarized as follows. 
(a) A large/small $\mathrm{Pe}$ means that the evaporation rate is lager/smaller than the rate of particle redistribution within the droplet. That is, a large/smaller $P e$ suggests relatively small particle mobility and the particles are included on the regressing droplet surface. As particle inclusion occurs, the droplet evaporation behavior departs from the classical $d^{2}-$ law for pure fluid droplet evaporation, as manifested by decreases in the effective area for evaporation $\left(A_{\text {eff }}\right)$, the evaporation constant $\left(K^{\prime}\right)$, and $\beta\left(\equiv K^{\prime} / K\right)$. The departure become more significant as $P e$ is increased.

(b) As $P e$ is increased, the departure from the $d^{2}$-law occurs earlier during the first evaporation stage and with a gradual decrease in $\beta$, compared with cases with smaller values of $P e$. Decreasing $P e$ causes more rapid decreases in $\beta$, which also occur toward the end of the first stage.

(c) For a given $Y_{\text {vo }}$, a large $P e$ leads to earlier shell formation with larger diameter $\left(d^{2} / d_{o}^{2}\right)$ than a small $P e$. For a given $P e$, larger/smaller values of $Y_{\text {vo }}$ lead to earlier/later formation of shell with lager/smaller diameters.

(d) For large aerodynamic diameter of dried particle congregates, a combination of large Pe and small $Y_{\mathrm{vo}}$ is preferred.

(e) Excessively large $Y_{\text {vo }}$ leads to very early shell formation and most of the evaporation takes place during the second stage, where liquid had to find its way (by such effects as capillary diffusion) to the droplet surface to evaporate. Because large $P e$ also promote early shell formation, the first stage evaporation exists on for $Y_{\mathrm{vo}} \cdot P e<1.8$.

(f) Given all other parameters fixed, $\theta$ also affects the evaporation rate constant. A smaller $\theta$ enables a larger degree of submerging included particle, leading to larger $A_{\text {eff }}$ and $\beta$ throughout the entire first stage.

Acknowledgment - Part of this work was conducted while RHC was on IPA assignment at the U.S. National Science Foundation (NSF) as a program director in the Division of Chemical, Bioengineering, Environmental, and Transport Systems, from 2012 to 2015). The support by NSF was through Individual Research Days. Both WD and RHC were previously with Department of Mechanical and Aerospace Engineering, University of Central Florida. 


\section{References}

[1] Maxwell, J.C., Collected Scientific Papers, Vol. 11, p. 625, Cambridge University Press, Cambridge (1890).

[2] H. G. Houghton, "A Study of the Evaporation of Small Water Drops," Physics, vol. 4, no. 12, p. 419 (1933).

[3] C. K. Law, "Recent advances in droplet vaporization and combustion," Prog. Energy. Combust. Sci., vol. 8, pp. 171-201 (1982).

[4] E. J. D. S.H. Zhang, "Mass Transfer from a single micro-droplet to a gas flowing at low Reynolds number," Chem. Eng. Comm., pp. 51-67 (1987).

[5] V. a. Kuz, "Evaporation of small drops," Journal of Applied Physics, vol. 69, no. 10, p. 7034 (1991).

[6] A. Lee and C. K. Law, "An experimental investigation on the vaporization and combustion of methanol and ethanol droplet," Combust. Sci. and Tech., vol. 86, pp. 253-265 (1992).

[7] D.B. Spalding, "The combustion of liquid fuels," Proceedings of the Combustion Institute, 4, 847-864 (1953).

[8] D.B. Spalding, Some Fundamentals of Combustion. Butterworth, London (1955).

[9] I. Glassman, R.A. Yetter, and N.G. Glumac (2015) Combustion, Fifth Ed., Chapter 5, Academic Press

[10] F.R. Newbold and N.R. Amundson, "A model for evaporation of a multicomponent droplet," AIChE journal vol. 19, no.1, 22-30 (1973).

[11] A. L. Yarin, G. Brenn, O. Kastner, and C. Tropea, "Drying of acoustically levitated droplets of liquidsolid suspensions: Evaporation and crust formation," Physics of Fluids, vol. 14, no. 7, p. 2289 (2002).

[12] W. E. Ranz and W. R. Marshall, JR, "Evaporation from drops," Chemical Engineering Progress, vol. 48, pp. 141-146 (1952).

[13] D. H. Charlesworth and W. R. Marshall, "Evaporation from drops contanining dissolved solids," AIChE Journal, vol. 6, no. 1, pp. 9-23 (1960).

[14] M. Mezhericher, A. Levy, and I. Borde (2010) Theoretical Models of Single Droplet Drying Kinetics: A Review; Drying Technology, 28: 278-293 (2010).

[15] M. Mezhericher, a. Levy, and I. Borde, "Modelling the morphological evolution of nanosuspension droplet in constant-rate drying stage," Chemical Engineering Science, vol. 66, no. 5, pp. 884-896 (2011).

[16] M. Mezhericher, M. Naumann, M. Peglow, a. Levy, E. Tsotsas, and I. Borde, "Continuous species transport and population balance models for first drying stage of nanosuspension droplets," Chemical Engineering Journal, vol. 210, pp. 120-135 (2012).

[17] D. Sen, S. Mazumder, J. S. Melo, A. Khan, S. Bhattyacharya, and S. F. D'Souza, "Evaporation driven self-assembly of a colloidal dispersion during spray drying: volume fraction dependent morphological transition.," Langmuir : the ACS journal of surfaces and colloids, vol. 25, no. 12, pp. 6690-6695 (2009).

[18] G. Derkachov, K. Kolwas, D. Jakubczyk, M. Zientara, and M. Kolwas, "Drying of a Microdroplet of Water Suspension of Nanoparticles: from Surface Aggregates to Microcrystal," Journal of Physical Chemistry C, vol. 112, no. 43, pp. 16919-16923 (2008).

[19] M. Mezhericher, a. Levy, and I. Borde, "Heat and mass transfer of single droplet/wet particle drying," Chemical Engineering Science, vol. 63, no. 1, pp. 12-23 (2008).

[20] R.-H. Chen, T. X. Phuoc, and D. Martello, "Effects of nanoparticles on nanofluid droplet evaporation," International Journal of Heat and Mass Transfer, vol. 53, no. 19-20, pp. 3677-3682 (2010).

[21] Y. Gan and L. Qiao, "Evaporation characteristics of fuel droplets with the addition of nanoparticles under natural and forced convections," International Journal of Heat and Mass Transfer, vol. 54, no. 2324, pp. 4913-4922 (2011).

[22] H. W. Cheong, G. V. Jefferys, and C. J. Mumford, "A receding interface model for the drying of slurry droplets," AIChE journal, vol. 32, no. 8, pp. 1334-1346 (1986).

[23] F. W. Abuaf, N., Staub, "Drying of liquid-solid slurry," Spray drying, vol. 1, pp. 277-284 (1987).

[24] S. Nesic and J. Vodnik, "kinetics of droplet evaporation," Chemical engineering science, vol. 46, no. 2, pp. 527-537 (1991). 
[25] X. D. Chen (2005) Air Drying of Food and Biological Materials-Modified Biot and Lewis Number Analysis, Drying Technology, 23: 2239-2248 (2005).

[26] P. Seydel, J. Blömer, and J. Bertling, "Modeling Particle Formation at Spray Drying Using Population Balances," Drying Technology, vol. 24, no. 2, pp. 137-146 (2006).

[27] C. S. Handscomb, M. Kraft, and a. E. Bayly, "A new model for the drying of droplets containing suspended solids," Chemical Engineering Science, vol. 64, no. 4, pp. 628-637 (2009).

[28] H. Minoshima, "Basic Model of Spray Drying Granulation.” pp. 472-478 (2001).

[29] H. Minoshima, K. Matsushima, L. H., and K. Shinohara, "Estimation of Dimaeter of Granule Prepared by Spray drying of slurry with fast adn easy evaporation," Journal of chemical engineering of Japan, vol. 35, no. 9, pp. 880-885 (2002).

[30] Dalmaz N, Ozbelge N, Eraslan HO, Uludag Y. "Heat and Mass Transfer Mechanisms in Drying of a Suspension Droplet: A New Computational Model," Drying Technology. 25(2): 391-400 (2007).

[31] Vehring R. 2008. Pharmaceutical Particle Engineering via Spray Drying. Pharmaceutical Research. 25(5): 999-1022 (2008).

[32] K. Sefiane, J. Skilling and J. MacGillivray, "Contact line motion and dynamic wetting of nanofluid solutions," Adv. Colloid and Interface Sci., 138:101-120 (2008).

[33] K. Sefiane and R. Bennacer, Nanofluids droplets evaporation kinetics and wetting dynamics on rough heated substrates, Advances in Colloid and Interface Science, 147-148, 263-271 (2009).

[34] F. Girard, M. Antoni, and K. Sefiane, On the Marangoni flow on evaporation rates of heated water drope, Langmuir, 24, 9207-9210 (2008).

[35]V. N. Paunov, "Novel Method for Determining the Three-Phase Contact Angle of Colloid Particles Adsorbed at Air-Water and Oil-Water Interfaces," Langmuir, no. 13, pp. 7970-7976 (2003).

[36] C. Zeng, H. Bissig, and a. D. Dinsmore, "Particles on droplets: From fundamental physics to novel materials," Solid State Communications, vol. 139, no. 11-12, pp. 547-556 (2006).

[37] C. Zeng, F. Brau, B. Davidovitch, and A. D. Dinsmore, "Capillary interactions among spherical particles at curved liquid interfaces," Soft Matter, vol. 8, no. 33, p. 8582 (2012).

[38] N. Tsapis, D. Bennett, B. Jackson, D. a Weitz, and D. a Edwards, "Trojan particles: large porous carriers of nanoparticles for drug delivery.," Proceedings of the National Academy of Sciences of the United States of America, vol. 99, no. 19, pp. 12001-5 (2002).

[39] J. Bahadur, D. Sen, S. Mazumder, S. Bhattacharya, H. Frielinghaus, and G. Goerigk, "Origin of buckling phenomenon during drying of micrometer-sized colloidal droplets.," Langmuir : the ACS journal of surfaces and colloids, vol. 27, no. 13, pp. 8404-14, (2011).

[40]R. Vehring, W. R. Foss, and D. Lechuga-Ballesteros, "Particle formation in spray drying," Journal of Aerosol Science, vol. 38, no. 7, pp. 728-746 (2007).

[41] K.H. Leong, "Morphological control of particles generated from the evaporation of solution droplets: theoretical considerations," Journal of Aerosol Science, vol. 18, no. 5, 511-524 (1987).

[42] W. J. Gerken, A. V. Thomas, N. Koratkar, M. A. Oehlschlaeger, "Nanofluid pendant droplet evaporation:experimens and modeling," Internationl Journal of Heat and Mass Transfer, vol. 74, 263-268 (2014).

[43] A. L. Yarin, G. Brenn, O. Kastner, D. Rensink, and C. Tropea, "Evaporation of acoustically levitated droplets," Journal of Fluid Mechanics, vol. 399, pp. 151-204 (1999). 